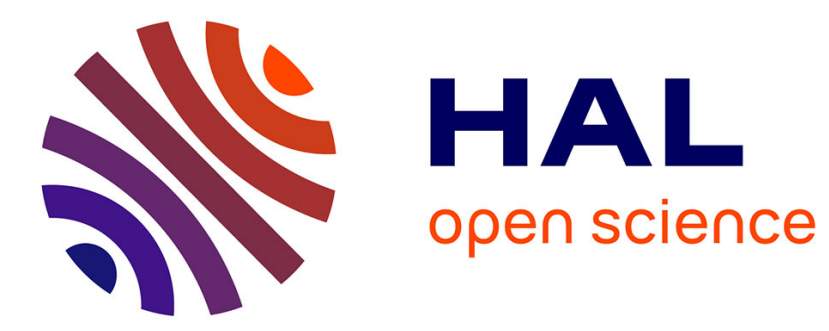

\title{
DÉCLINAISONS DU MONOTHÉISME
}

\author{
Fethi Benslama
}

\section{To cite this version:}

Fethi Benslama. DÉCLINAISONS DU MONOTHÉISME. Cliniques méditerranéennes, 2006, 1 (73), pp.5 - 8. 10.3917/cm.073.0005 . hal-01502402

\section{HAL Id: hal-01502402 \\ https://hal.science/hal-01502402}

Submitted on 5 Apr 2017

HAL is a multi-disciplinary open access archive for the deposit and dissemination of scientific research documents, whether they are published or not. The documents may come from teaching and research institutions in France or abroad, or from public or private research centers.
L'archive ouverte pluridisciplinaire HAL, est destinée au dépôt et à la diffusion de documents scientifiques de niveau recherche, publiés ou non, émanant des établissements d'enseignement et de recherche français ou étrangers, des laboratoires publics ou privés. 


\section{DÉCLINAISONS DU MONOTHÉISME}

\section{Argument}

Fethi Ben Slama

\section{ERES | «Cliniques méditerranéennes »}

$2006 / 1 \mathrm{n}^{0} 73 \mid$ pages 5 à 8

ISSN 0762-7491

ISBN 2-7492-0591-3

Article disponible en ligne à l'adresse :

http://www.cairn.info/revue-cliniques-mediterraneennes-2006-1-page-5.htm

\section{Pour citer cet article :}

Fethi Ben Slama, «Déclinaisons du monothéisme. Argument », Cliniques méditerranéennes 2006/1 ( $\left.\mathrm{n}^{0} 73\right)$, p. 5-8.

DOI 10.3917/cm.073.0005

Distribution électronique Cairn.info pour ERES.

(C) ERES. Tous droits réservés pour tous pays.

La reproduction ou représentation de cet article, notamment par photocopie, n'est autorisée que dans les limites des conditions générales d'utilisation du site ou, le cas échéant, des conditions générales de la licence souscrite par votre établissement. Toute autre reproduction ou représentation, en tout ou partie, sous quelque forme et de quelque manière que ce soit, est interdite sauf accord préalable et écrit de l'éditeur, en dehors des cas prévus par la législation en vigueur en France. Il est précisé que son stockage dans une base de données est également interdit. 


\title{
Fethi Benslama
}

\section{Déclinaisons du monothéisme Argument}

\author{
« De la psychanalyse, qui la première a mis \\ à découvert la surdétermination régulière des actes \\ et formations psychiques, on n'a pas à craindre \\ qu'elle soit jamais tentée de faire dériver \\ d'une origine unique quelque chose d'aussi compliqué \\ que la religion. Alors que, dans une unilatéralité \\ nécessaire, à vrai dire conforme à son devoir, \\ elle veut faire reconnaître une seule des sources \\ de cette institution, au départ elle ne revendique \\ pour celle-ci pas plus l'exclusivité que le premier rang \\ parmi les facteurs agissant conjointement. \\ Seule une synthèse à partir de divers domaines \\ de recherche pourra décider quelle significativité relative \\ il faut attribuer dans la genèse de la religion \\ au mécanisme dont il sera discuté ici ; \\ mais un tel travail dépasse aussi bien les moyens \\ que la visée du psychanalyste. » \\ S. Freud, Totem et tabou, OC., XI, p. 311.
}

Cet avertissement de Freud, venant au début du dernier chapitre de Totem et tabou, ne mériterait pas d'être rappelé aujourd'hui, s'il ne prenait une acuité particulière quant à l'approche que les psychanalystes sont susceptibles de mettre en œuvre devant l'actualité du fait religieux. Tout en soulignant la légitimité et la nécessité du point de vue psychanalytique, en l'occurrence sa reconnaissance de la source infantile de la religiosité et de son noyau de désirance (Sehnsucht) pour le père, il en appelle aux autres domaines de recherche pour le situer dans sa portée significative. Ainsi, la dérivation « d'une origine unique » de la religion dans les limites de la psycha-

Fethi Benslama, psychanalyste, professeur de psychopathologie à l'Université Paris 7, 26 rue de Paradis, 75011 Paris ; 7 rue Pinel, 93200 Saint-Denis. 
nalyse n'est envisageable que parce qu'elle est sous-tendue par la "surdétermination » et par " la complexité », et qu'elle doit se confronter à la relativité des mécanismes. En somme, l'audace de l'hypothèse reste toujours veillée par la conscience de l'hétérogène. C'est sous cette enseigne que nous aimerions engager une réflexion sur la situation des religions monothéistes dans le monde actuel, et faire état des recherches référées à la psychanalyse et aux champs limitrophes qui se sont développés au cours des dernières années.

Ce préliminaire implique qu'il n'y a pas à désigner un lieu plus approprié ou plus adéquat (de " premier rang », dit Freud) à partir duquel $L a$ religion, ou Le monothéisme puisse se donner à penser au regard de l'excès, de l'irréductibilité ou de l'hétérogénéité qu'ils présentent ; même pas la question de l'Un, puisqu'elle est toujours l'objet d'une équation qui, pour ne s'en tenir qu'aux monothéismes abrahamiques, se manifeste dans le judaïsme par la profération du buisson ardent (« Je suis qui Je suis»), dans le christianisme par l'Un en trois Personnes, et en islam par une formulation négative («pas d'autre divinité que Lui »). C'est bien pourquoi, ces quelques lignes ne prétendent pas à autre chose qu'à indiquer, et seulement indiquer, quelques parages pour partager une réflexion sur le monothéisme, appelée ici comme rapport à des déclinaisons, non sans songer à tout ce qui se réserve dans le radical de ce mot : de l'écart, de la dérivation, de l'inclination, voire du déclin, ou bien encore des verbes de parole : dire, composer, réciter.

Avant d'aller vers l'actualité de la religion, notons que cette invitation s'introduit par la question de la dénomination et de l'innommable juif, chrétien, musulman. Cet engagement n'est pas incident, il signale que dans ces trois lieux, le monothéisme est sous-tendu par un rapport au langage qui a rompu l'adéquation entre le nom et l'essence du divin, c'est-à-dire l'essentialité même. Du reste, c'est à ce point que Lacan reprend l'élaboration freudienne autour de Moïse, et lui donne des développements théoriques importants. Plus récemment et dans le même sens, l'égyptologue Jan Assmann a publié une étude (Moïse l'Égyptien, Aubier, 2001) dans laquelle il montre comment « la distinction mosaïque » a consisté en un coup d'arrêt à l'inter-traductibilité des dieux dans le cosmothéisme. Le monothéisme se fonderait ainsi sur un refus de la traduction qui cristallise une mutation dans la civilisation où le langage n'assure plus une communication médiate entre les mots et les choses. Comment penser l'institution de ce " défaut de traduction » (qui est pour Freud, rappelons-le, l'équivalent du refoulement), tout à la fois sur le plan de la structure de la subjectivité, et dans l'ordre général de la civilisation monothéiste ? Quels sont les effets de cet intraduisible à l'intérieur de chacune des religions dites « révélées » et entre elles?

Si l'on considère le phénomène de la résurgence du « religieux » dans sa portée mondiale, nous n'en sommes plus aujourd'hui à le considérer comme 
un simple " retour de... », ainsi qu'on l'a prétendu trop rapidement à ses débuts. Il faut bien constater que les demandes et les forces qui le manifestent entrent en composition avec des dimensions extérieures à la religion, du moins telle qu'on croit la définir, dont de toute évidence le politique (le nationalisme), le biologique (la logique de la représentation de la vie), la technique (la machine télé-technoscientifique), ou l'économie comme mouvement de radicalisation du fétichisme ; au point que les distinctions entre le politique et le religieux, la religion et la science s'en trouvent brouillées dans les discours. S'agit-il là des avatars de la décomposition de la religion, ou bien plus radicalement (et peut-être en même temps) de la venue à échéance d'oppositions et de clivages tels que foi et savoir, raison et religion, croyance et science dont les antagonismes et les surenchères dérobent l'unité de leur source (J. Derrida, Foi et savoir, Le Seuil, 1996) ? Ces considérations conduisent à rouvrir les questions : qu'est-ce que croire ?, qu'appelle-t-on religion (quid de tout ce lexique) ?, auxquelles la pensée psychanalytique ne peut rester indifférente, puisqu'il y va du rapport de ses concepts, au temps. Dans la même optique, nous ne pouvons manquer de reposer le problème des sources, des ressources, des origines de la croyance (que l'exergue soulève précisément), des multiples lieux de son expérience quand s'y nouent le « croire » et le « savoir».

Dans ce contexte, l'irruption de l'islam sur la scène actuelle du monde, a fait émerger des questions inédites, qui dépassent le domaine réservé de l'islamologie. En une décade, le champ des sciences de l'homme et de la culture s'en est fait l'écho, sous l'effet d'une urgence à penser ce qui arriva à l'islam et arriva de son tumulte au monde. Ce fait est d'autant plus notable que l'islam est resté pour une large part en marge des foyers de l'épistémè moderne, à l'écart des modalités que la rationalité laïque avait inventés dans ses rapports avec le judaïsme et le christianisme. Marge et écart ont fini par engendrer une forme d'agnosie concernant la dernière religion monothéiste. À l'instar d'autres disciplines, la psychanalyse ne fait pas exception à cette situation. Mise à part une mention rapide chez Freud dans L'homme Moïse et la religion monothéiste, les études psychanalytiques sont restées muettes sur " le cas de la fondation de la religion mahométane " (expression de Freud). En même temps, on ne peut manquer de relever et d'interroger la lacune relative à la psychanalyse dans la réception des savoirs sur l'homme, dans le monde musulman. Toutefois, ici comme là, la dernière décade a vu se défaire partiellement cette imperitia réciproque, de sorte que l'on peut se demander si nous ne sommes pas à l'époque de la déconstitution d'une double agnosie, qui permet d'envisager à la lueur de son événement, l'ensemble des déclinaisons du monothéisme sous un autre jour. 
La réflexion sur le monothéisme ne peut laisser de côté la question de la violence dont il prétend organiser l'économie symbolique. Le caractère sacré de la vie s'y affirme en même temps que la nécessité du sacrifice, sans que « la révélation de la parole » puisse rompre ce cercle. Existe-t-il une violence propre au monothéisme, ou/et proprement religieuse ? Jusqu'à quel point peut-on parler de guerre de religion et de guerre supposée ne pas l'être (au nom de la raison du droit national, international, humanitaire, etc.) ? En quel sens y a-t-il lieu de distinguer encore entre violence divine et violence mythique (W. Benjamin), quand la rationalité hyper-technique les rapproche au point que nous constatons aujourd'hui ? Sommes-nous à un nouvel âge de la cruauté ? Comment, du point de vue de la logia de l'inconscient, la pensée psychanalytique se saisit-elle de la répétition, de la mutation ou de l'inédit sur ce plan ? À quelles transformations problématiques cela est-il susceptible de conduire, au regard de la clinique et de la théorie métapsychologique ? 